\title{
The effect of NAFLD (non-alcoholic fatty liver disease) on long-term outcome of chronic hepatitis B in Iranian patients
}

\author{
Arezoo Estakhri ${ }^{1}$, Ali Akbari Sari ${ }^{2}$, Sahar Naz Nedjat ${ }^{2}$, Marym Rohban $^{1}$, Naser Rakhshani ${ }^{1}$, \\ Seyed Mohammad Tavangar ${ }^{1}$, Reza Malekzadeh ${ }^{1}$, Ghodrat Montazeri ${ }^{1}$ \\ ${ }^{1}$ Digestive Disease Research Center (DDRC), Shariati Hospital, Tehran University of Medical Sciences, Tehran, Iran
${ }^{2}$ School of Public Health, Knowledge Utilization Research Center, Tehran University of Medical Sciences, Tehran, Iran
Email: arezooestakhri@yahoo.com
}

Received 19 November 2011; revised 21 December 2011; accepted 5 January 2012

\begin{abstract}
Background: The influence of Non-Alcoholic Fatty Liver Disease on the outcome of chronic hepatitis B disease, including viral, biochemical and histologic characteristics, in Iranian patients is not yet fully understood. Aim: To evaluate the effect of Non-Alcoholic Fatty Liver Disease (NAFLD) on long-term histologycal, biochemical and viral outcome of chronic hepatictis $B$ in Iranian patients. Methods: We retrospectively evaluated 94 "e Ag" negative chronic hepatitis B patients (with NAFLD: 44, without NAFLD: 50). Non-Alcoholic Fatty Liver Disease was diagnosed based on liver biopsy according to Kleiner classification. Liver biopsy was done for all patients. Serological and biochemical variables were evaluated with repeated measure analysis. Results: Non-Alcoholic Fatty Liver Disease (NAFLD) was present in $47 \%$ of the patients (44 out of 94 patients). In the NAFLD group, increase in AST, ALT, stage ( $P=0.002)$, grade, and total score of liver biopsy were independently related to non-alcoholic fatty liver disease, while HBV-DNA viral load did not correlate with the presence of a fatty liver. Conclusion: Abnormalities of liver enzymes and liver histopathology are more prevalent in concurrent chronic hepatitis $B$ and Non-Alcoholic Fatty Liver Disease (NAFLD).
\end{abstract}

Keywords: Non-Alcoholic Fatty Liver Disease; Chronic Hepatitis B; IMPACT; Long-Term Prognosis; Iranian

\section{INTRODUCTION}

Hepatitis B virus (HBV) infection is an important health concern that can lead to cirrhosis and hepatocellular carcinoma, both with a high morbidity and mortality rate $[1,2]$. In Chronic hepatitis B, there are some agents that can have a significant role in progression to cirrhosis, such as age, sex, co-infection with hepatitis D, high levels of ALT, high HBV-DNA viral load, HBeAg status and HBV genotype [3].

Moreover, of the above-mentioned causes, it seems that Non Alcoholic Fatty Liver Disease (NAFLD)-a chronic liver disease that is defined by lipid droplets in more than 5\% of hepatocytes [4] and characterized by diffuse fatty inflammation and infiltration-is also a reason for leading to advanced liver disease in almost $15 \%$ $20 \%$ of the patients [5]; however, its prevalence differs in different patient populations and geographical locations [6]. It is estimated that its prevalence is $20 \%$ in the general population, ranging from $15 \%$ to $39 \%$. NAFLD is the most common liver disease in the United States [7].

It has been shown that the frequency of chronic heaptitis B is $27 \%-51 \%$, while chronic hepatitis C has a frequency between 31 and $72 \%$ [8].

In particular, steatosis is now well-recognized as a factor that accelerates with the development of fibrosis in chronic hepatitis C. In other words, livers with fatty changes are more vulnerable to be fibrosed and inflamed when they are faced with other liver diseases. There are many studies regarding the effect of NAFLD on the progression of chronic hepatitis $\mathrm{C}$ disease [9-11].

However, there is little data on the co-existence of NAFLD with chronic hepatitis B and the outcome of this chronic viral liver disease. In this study, we aimed to evaluate the effect of NAFLD on long-term prognosis of chronic hepatitis $\mathrm{B}$.

\section{PATIENTS AND METHODS}

\subsection{Patients}

Ninety four chronic hepatitis B patients who were referred to the Digestive Disease Research Centre (DDRC) of Shariati Hospital from Jan 2006 to Jan 2009 were enrolled in a nested case-control study. The mean \pm SD of 
age was $43.24 \pm 12.22$ years. Patients with other liver diseases such as hepatitis $\mathrm{C}$ and autoimmune hepatitis were excluded from the study.

The diagnosis of chronic hepatitis B was established through the positivity of hepatitis B surface antigen for more than 6 months, elevated alanine aminotransferase levels and serum HBV-DNA viral loads.

To investigate the effect of non alcoholic fatty liver disease on the prognosis of chronic hepatitis $B$, these patients were divided into 2 groups of with and without non-alcoholic fattyliver disease, according to the findings of liver biopsy.

Biochemical, virologic and pathologic factors, and liver enzymes levels were measured every 3 months from the beginning of the study, while measurement of HBV-DNA viral load was done every 6 months using TaqMan RealTime Polymerase Chain Reaction assay with a dynamic range of quantification from 54 to $1.1 \times 108 \mathrm{IU} / \mathrm{ml}(1.7$ to $8.0 \log 10 \mathrm{IU} / \mathrm{ml}$ ) [12].

Liver biopsy was performed 2 times, at the beginning and at the end of the study. Then, histological analysis of the liver sections was reviewed by two hepatopathologists who were blinded to the clinical data [13]. The presence of steatosis was diagnosed according to the Kleiner's classification [13]. This scoring system consists of the following: percentage of macrovesicular steatosis (0 - 3), lobular inflammation (0 - 3), ballooning degeneration $(0-2)$, stage $(0-4)$ and portal inflammation (0 - 1). In the classification of steatosis, less than $5 \%$, $5 \%$ - 25\% and more than 25\% steatosis were considered normal, mild, and severe fat deposition, respectively
[13]. Moreover, other related factors Such as age, gender and BMI were evaluated, as well.

\subsection{Statistical Analysis}

Data was analyzed with SPSS (Inc., Chicago, IL, USA) software.

The results of quantitative and qualitative variables were presented as mean \pm SD and percentages, respecttively.

Moreover, in order to assess the trend of virological, biochemical and histological markers of all patients and each subject in the NAFLD group and compare them with the control group, repeated measure analysis was used.

For all comparisons, $\mathrm{P}$ value $<0.05$ was considered significant.

\section{RESULTS}

\section{Cases and Controls}

Ninety four patients with chronic hepatitis B (CHB) were enrolled in our study. Then, based on the existence of non-alcoholic fatty liver disease (NAFLD), subjects were divided into two groups: forty-four patients with NAFLD in the case group and fifty patients without NAFLD in the control group. NAFLD was confirmed by liver biopsy, which was available and done for all patients. HBV genotype was D in all patients [14]. Demographic and biochemical characteristics of the patients are shown in Tables 1 and 2.

Table 1. Demographic characteristics of the patients.

\begin{tabular}{lcc}
\hline \multicolumn{1}{c}{ Characteristics } & Cases & Controls \\
\hline \multirow{2}{*}{ Men } & $(\mathrm{n}=44)(\%)$ & $(\mathrm{n}=50)(\%)$ \\
Women & $33(75 \%)$ & $39(78 \%)$ \\
Mean age (years) \pm SD & $11(25 \%)$ & $11(22 \%)$ \\
& $46.07 \pm 11.10$ & $40.76 \pm 12.73$ \\
Not overweight $(15-24.9)$ & Body Mass Index (BMI) & \\
Overweight (25 - 29.9) & $27.3 \%$ & $44 \%$ \\
Obese (30 - 50) & $36.4 \%$ & $12 \%$ \\
\hline
\end{tabular}

Table 2. Biochemical characteristics of the patients.

\begin{tabular}{cccc}
\hline Characteristics & Cases & Controls & P-value \\
\hline & $(\mathrm{n}=44)$ & $(\mathrm{n}=50)$ & \\
AST & $41.45 \pm 2.91$ & $37.37 \pm 2.72$ & 0.01 \\
ALT & $57.95 \pm 4.37$ & $45.97 \pm 4.09$ & 0.007 \\
HBV-DNA (log copies/ml) & $2.41 \pm 1.87$ & $3.44 \pm 1.76$ & 0.1 \\
Genotype & $\mathrm{D}$ & $\mathrm{D}$ & 0.002 \\
Stage & $1.67 \pm 1.3$ & $1.2 \pm 1.18$ & 0.09 \\
Grade & $5.25 \pm 2.24$ & $4.77 \pm 2.00$ & \\
\hline
\end{tabular}


In the NAFLD group, during 3 years, AST (Figures 1 and 2) was sometimes in the normal range while ALT was always above the normal limit. It is important to note that the trend of both AST and ALT in the NAFLD group was higher than the control group (AST: $41.45 \pm$ 2.91 vs. $37.37 \pm 2.72$ and ALT: $57.95 \pm 4.37$ vs. $45.97 \pm$ 4.09). Regarding liver histopathology (Figures 3 and 4), the stage and the grade scores were higher in cases than controls $(1.67 \pm 1.3$ vs. $1.2 \pm 1.18)$. During the 3 years, stage significantly increased $(\mathrm{P}=0.002)$, while grade decreased insignificantly $(\mathrm{P}=0.091)$. Just the same, Westin et al. found that the progression of fibrosis on follow-up liver biopsies was more prevalent in patients with steatosis in the first biopsy [15]. NAFLD had no impact on HBV-DNA level (Figure 5). In other words, the level of HBV-DNA in the case group was lower than the control group $(2.41 \pm 1.87$ vs. $3.44 \pm 1.76)$ at the end of the study while it was very high in the beginning of the study ( $>100,000$ vs. $<20,000$ copy/ml).

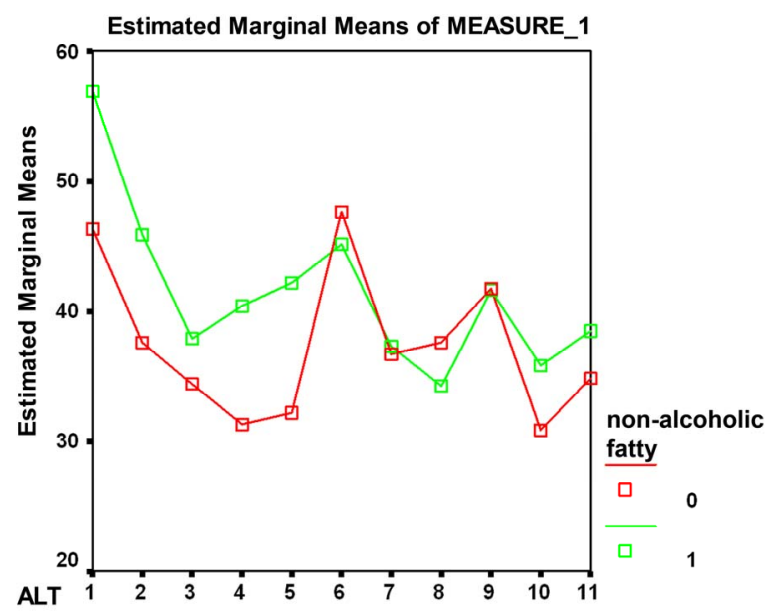

Figure 1. Range of AST.

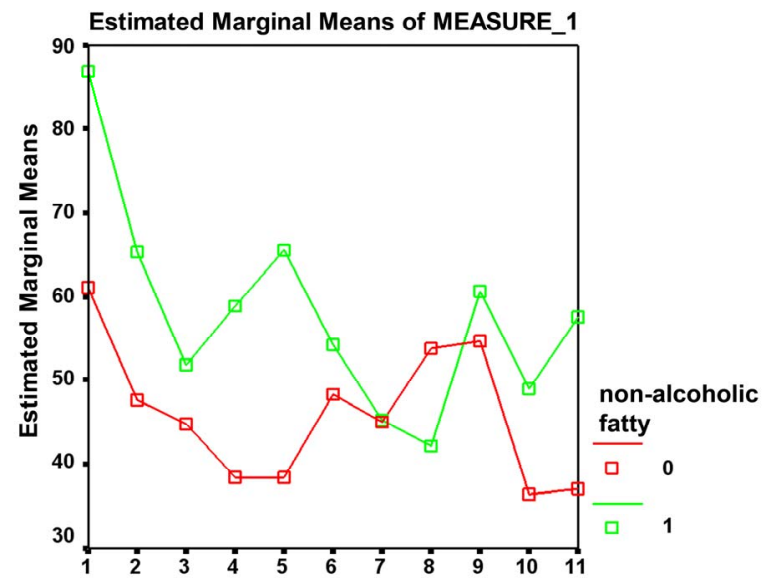

ALT

Figure 2. Range of ALT.

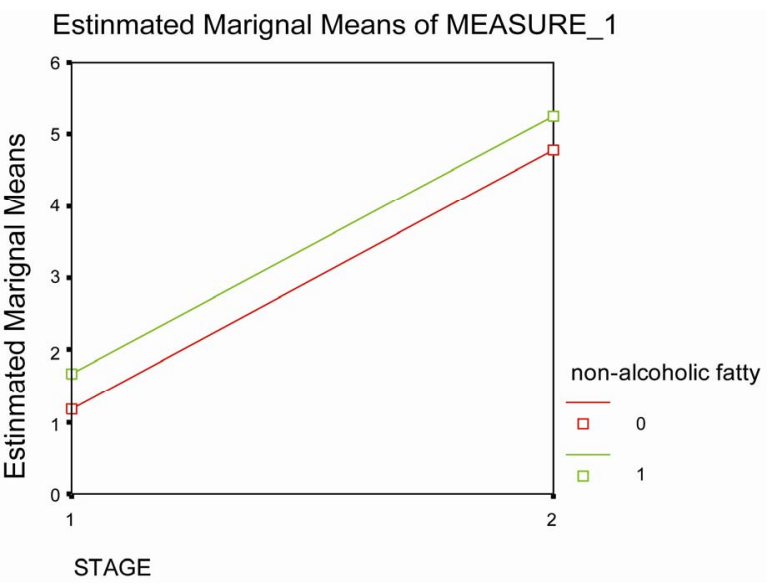

Figure 3. Liver histopathology stage 1.

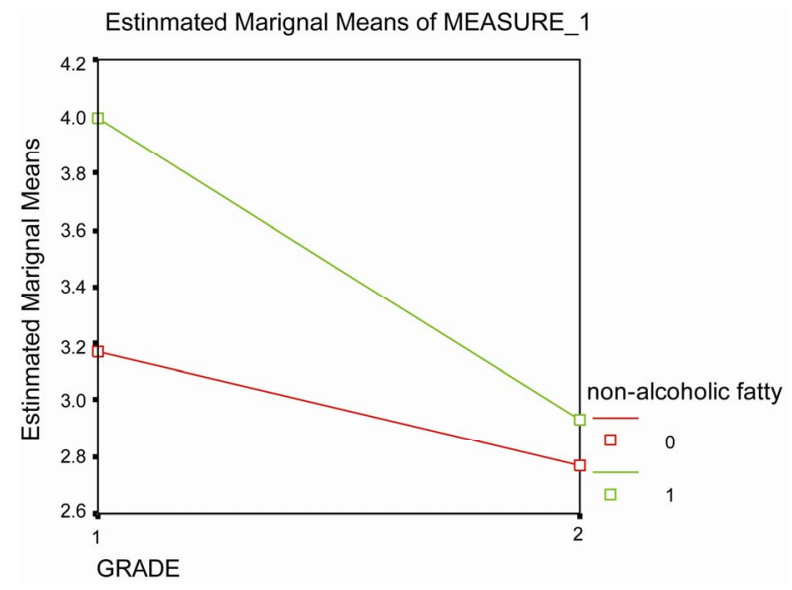

Figure 4. Liver histopathology stage 2.

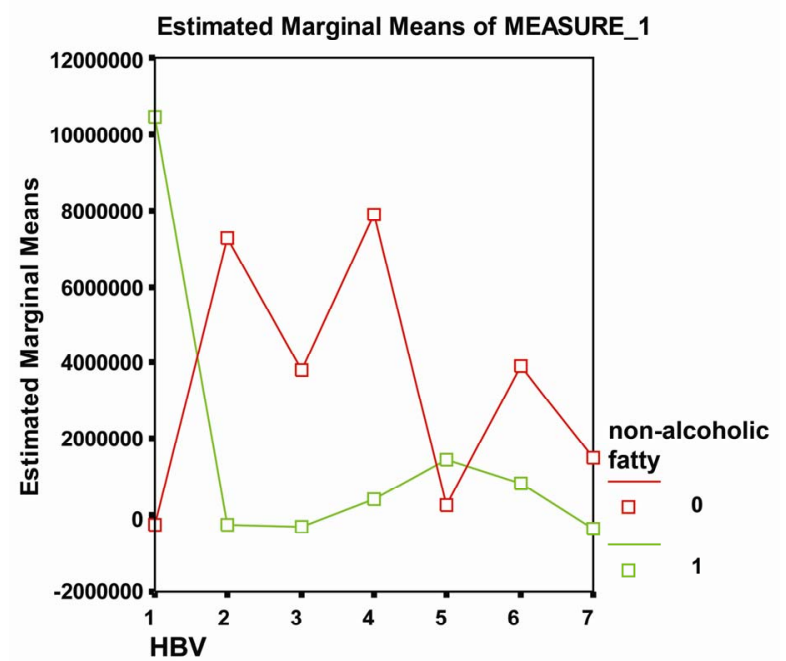

Figure 5. NAFLD had no impact on HBV-DNA level.

\section{DISCUSSION}

In this study, we tried to investigate the effect of NonAlcoholic Fatty Liver Disease (NAFLD) on histologic, 
viral and biochemical characteristics of chronic hepatitis B.

Non Alcoholic Fatty Liver Disease (NAFLD) is a metabolic disorder which affects the liver parenchyma, leading to liver cirrhosis.

It has been shown that hepatic steatosis is present in more than $30 \%-70 \%$ of the patients with chronic hepatitis $\mathrm{C}$ and that NAFLD can accelerate liver damage. In such patients, the prognosis and response to anti-viral medications like Ribavirin and Peginterferone is poor [16].

Also, it has been documented that a fatty liver makes patients more vulnerable to other liver injury factors, facilitating fibrosis and inflammation in chronic hepatitis $\mathrm{C}$ [17].

However, data regarding the concurrent chronic hepatitis B and non-alcoholic fatty liver disease is limited.

Our findings showed that components of chronic HBV, including liver enzymes and liver histology (stage and grade), were influenced by NAFLD while HBV-DNA viral load showed no correlation with NAFLD existence as both the aforementioned factors increased during the three years of study. In other words, HBV-DNA viral load had no relationship with NAFLD.

Our study also showed that obesity (BMI > 30) was more frequently seen in the NAFLD group compared to the control group ( $36.4 \%$ vs. $12 \% \mathrm{~kg} / \mathrm{m}^{2}, \mathrm{P}=0.02$ ), meaning that hepatic steatosis in NAFLD patients is probably due to host factors rather than viral factors $[18,19]$.

Also, our analysis showed no significant interaction between liver enzymes (AST, ALT) and age or BMI.

In summary, concurrent NAFLD and chronic hepatitis $\mathrm{B}$ can lead to liver fibrosis and inflammation and result in abnormal liver enzymes, especially ALT.

\section{ACKNOWLEDGEMENTS}

The authors gratefully acknowledge and thank Dr. Reza MalekzadehHead of Digestive Disease Research Center (DDRC), Shariati Hospital, Tehran, Iran—in this study.

\section{REFERENCES}

[1] Bondini, S. and Kallman, J. (2007) Impact of non-alcoholic fatty liver disease on chronic hepatitis B. Liver International, 27, 607-611. doi:10.1111/j.1478-3231.2007.01482.x

[2] Ganem, D. and Prince, A.M. (2004) Hepatitis B virus infection-natural history and clinical consequences. The New England Journal of Medicine, 350, 1118-1129. doi:10.1056/NEJMra031087

[3] Tran, T.T. and Martin, P. (2004) Hepatitis B: Epidemiology and natural history. Clinical Liver Disease, 8, 255266. doi:10.1016/j.cld.2004.02.008

[4] Altiparmak, E. and Koklu, S. (2005) Viral and host causes of fatty liver in chronic hepatitis B. World Journal of
Gastroenterology, 11, 3056-3059.

[5] Stephen, A. and Harrison, M. (2002) Nonalcoholic steatohepatitis: What we know in the new Millennium. The American Journal of Gastroenterology, 97, 2714-2724.

[6] Tsang, S.W.C. and Ng, W.F. (2006) Predictors of fibrosis in Asian patients with non-alcoholic steatohepatitis. Journal of Gastroenterology and Hepatology, 21, 116-121. doi:10.1111/j.1440-1746.2005.04146.X

[7] Younossi, Z.M. and Deihl, A.M. (2000) Nonalcoholic fatty liver disease: An agenda for clinical research. Hepatology, 35, 746-752. doi:10.1053/jhep.2002.32483

[8] Gordon, A. and McLean, C.A. (2005) Hepatic steatosis in chronic hepatitis B and C: Predictors, distribution and effect on fibrosis. Journal of Hepatology, 43, 38-44. doi:10.1016/j.jhep.2005.01.031

[9] Lonardo, A. and Adinolfi, L.E. (2004) Steatosis and hepatitis C virus: Mechanismsm and significance for hepatic and extrahepatic disease. Gastroenterology, 126, 586597. doi:10.1053/j.gastro.2003.11.020

[10] Ong, J.P. and Younossi, Z.M. (2001) Chronic hepatitis C and superimposed nonalcoholic fatty liver disease. Liver, 21, 266-271. doi:10.1034/j.1600-0676.2001.021004266.x

[11] Adinolfi, L.E. and Gambardella, M. (2001) Steatosis accelerates the progression of liver damage of chronic hepatitis $\mathrm{C}$ patients and correlated with specific HCV genotype and visceral obesity. Hepatology, 33, 1358-1364. doi:10.1053/jhep.2001.24432

[12] Chevaliez, S. and Bouvier-Alias, M. (2008) Performance of the cobas ampliprep/cobas taqman real-time PCR assay for hepatitis B virus DNA quantification. Journal of clinical Microbiology, 46, 1716-1723. doi:10.1128/JCM.01248-07

[13] Kleiner, D.E. and Brunt, E.M. (2005) Design and validation of a histological scoring system for nonalcoholic fatty liver disease. Hepatology, 41, 1313-1321. doi:10.1002/hep.20701

[14] Bahramali, G. and Sadeghizadeh, M. (2008) Clinical, virological and phylogenetic features of hepatitis Binfection in Iranian patients. World Journal of Gastroenterology, 14, 448-453

[15] Westin, J. and Nordlinder, H. (2002) Steatosis accelerates fibrosis development over time in hepatitis c virus genotype 3 infected patients. Journal of Hepatology, 37, 837842. doi:10.1016/S0168-8278(02)00299-4

[16] Wong, G.L.-H. and Wong, V.W.-S. (2009) Metabolic syndrome increases the risk of liver cirrhosis in chronic hepatitis B. Gut, 58, 111-117. doi:10.1136/gut.2008.157735

[17] Powell, E.E. and Jonsson, J.R. (2005) Steatosis: Co-factor in other liver diseases. Hepatology, 42, 5-13. doi:10.1002/hep.20750

[18] Thomopoulos, K.C. and Arvaniti, V. (2006) Prevalence of liver steatosis in patients with chronic hepatitis B. European Journal of Gastroenterology \& Hepatology, 18, 233237. doi:10.1097/00042737-200603000-00002

[19] Altlparmak, E. and Koklu, S. (2005) Viral and host causes of fatty liver in chronic hepatitis B. World Journal of Gastroenterology, 11, 3056-3059. 